\title{
Research on the Cultural Product Design Based on Consumer Cognition
}

\author{
Jianxin Cheng, Junnan Ye, Le Xi, and Wangqun Xiao \\ School of Art, Design and Media, East China University of Science and Technology \\ M. BOX 286, NO. 130, Meilong Road, Xuhui District, Shanghai 200237, China \\ cjx.master@gmail.com, yejunnan971108@qq.com, xilutar@sina.com, \\ xiaoyao-1916@163.com
}

\begin{abstract}
With the development of cultural creative industry, cultural product design has shifted from "production-oriented" of batch design to "consumeroriented" of personalized design, on the basis of function, shape, material, color, brand, economic, cultural and other factors of product design. The consumer's emotional appeal should also be investigated fully and comprehensively. How to master the general rule of consumers' psychology, follow the law of consumer behavior, design marketable products and finally promote the customers' satisfaction will be significant subjects of current cultural product design.

This paper studies on the current cultural product from targeted consumers' cognition point of view based on theories such as Demand Psychology, Consumption Psychology, Cognitive Psychology, Kansei Engineering, Ergonomics, Semantics of Design and Semiotics and etc, and proposes three hierarchy theory of consumer cognition on cultural product by analyzing cultural products' connotation, characteristic and category and combining human cognitive system in Cognitive Psychology, which are interpretation, experience and cognition. Also present the cultural product design procedure based on consumer cognition on the basis of this theory, including research and analysis of consumers' cognition, image space establishment of cultural product cognition, cultural product concept design and implementation and cultural product test and evaluation.
\end{abstract}

Keywords: Consumer Cognition, Cultural Product Design, Design Method, Design Procedure.

\section{Introduction}

With the development of social economy, people are not satisfied with the material enjoyment of modern products, and try to pursue the intrinsic cultural connotation and artistic value, which change people's material life, improve their life quality and bring people with higher spiritual enjoyment, making the world much more wonderful. Therefore, cultural influence on product design should be strengthened; unique cultural quality should be added to the products so as to show products' unique style and charm, which could make product design in a dominant position in international market competition. Liu,2009). 
Designers must learn to cater to the desire of consumers from user's point of view, make the shape of product meet their emotional demand. They should realize the emotional communication between people and product in the designation and make the product not only satisfy people's living needs, but also an emotion-bearing companion (P. M. A. Desmet. C. J. Overbeeke. S. J. E. T. Tax,1993).

Thus how to master the general rule of consumers' psychology, follow the law of consumer behavior, design marketable products and finally promote the customers' satisfaction will be significant subjects of current cultural product design.

\section{Research Background}

\subsection{Cultural Products' Ponnotation, Characteristic and Category}

In the latest document "1994-2003, the International Flow of Cultural Products and Services" released by UNESCO, it said that cultural products are the products provided by culture industry activities, which are divided into two categories of cultural products and cultural service (li,2013).

Professor Bai Ying in Central South University thinks cultural products have the following characteristics: first, it reflects in the creativity of symbols in pragmatic sense used by communicators; second, it reflects in the value deferring by added value of symbolic values; third, the cultural resources regeneration or proliferation regurgitation-feeding that formed by value deferring; fourth, the ideology of significant social and cultural responsibility by symbolic interaction (Bai,He,2006).

Sun Anmin divides cultural products into three categories: art, handicrafts, and industrial products. Art is personalized, created by a single person, such as drama, calligraphy, and painting; handicrafts is done manually by some person or persons, such as tourism dance, sculpture; industrial products are standardized and can carry out mass production, such as television, DVDs, books and magazines (Sun Anmin, 2005).

\subsection{Consumer Cognitive Model}

Cognitive system in the psychology is made up of four parts: the information transmission and processing system of limited capacity, cognitive system, knowledge system and self -monitoring system.

Modern cognitive psychology thinks that the human brain is an information processing system. When people are awake, they will continue to carry out processing activities from the stimulation of external environment information. This system mainly includes the following components: sensors, registration, pattern recognition, short-term memory and long-term memory (Zhang, 2007).

\section{$3 \quad$ Research Methods}

\subsection{Three Levels of Consumers' Cognition on Cultural Product}

Consumers' cognition for cultural products is based on their cognitive model, and in this process, we can include the consumers' cognition process into three levels, namely, interpretation, experience, and cognition, as shown in Figure 1. 


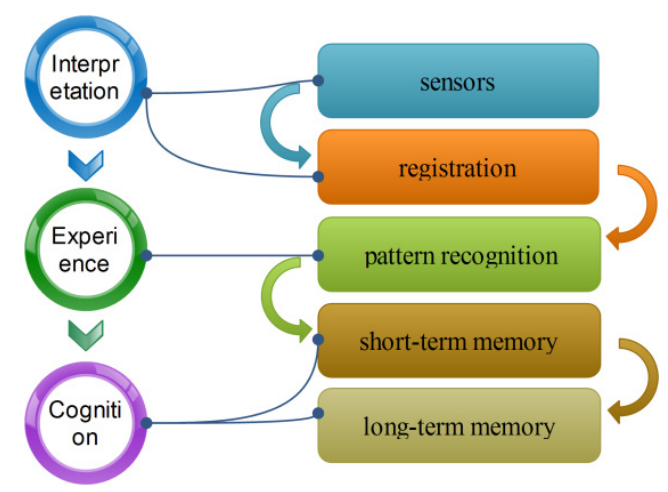

Fig. 1. Three Levels of Consumers' Cognition for Cultural Products

\section{Interpretation}

Interpretation is the receptor and sensory register part in cognitive models of consumers where consumers can do cognitive experience of initial level on properties such as cultural products' color, texture, shape, surface ornamentation, detail processing, and component through the visual, tactile, auditory, olfactory, and gustatory sense .

\section{Experience}

Experience is a part of recognition pattern in consumers' cognitive model, and in the process consumers, according to their social, cultural, economic, political environment, and their own cultural cognition, have the deep experience in cultural products ,which means the deeply-cognized experience in attributes such as the process, function, operation, safety, and relationship .

\section{Cognition}

Cognition is a short-term memory and long-term memory part in consumers' cognitive model .In the process of interpretation and experience of cultural products, consumers understand properties of products such as a special meaning intrinsically expressed, narrative, emotionality, and cultural characteristics, and stored internal information of these products in short-term memory. After repeating product experience cognition, consumers formed a cultural stereotype in their thinking set, so as to achieve the long-term memory.

\subsection{Access Management Product Design Program}

For the product design procedure, Canada's new product development expert Robert G.Copper proposed the "gateway management procedures" in 1993. Now the design management process has been adopted by many international well-known large companies, including familiar Carlsberg public limited company, Kodak company, Lego company, Microsoft Corp, Hewlett-Packard Co, DuPont Co and many other wellknown enterprises, which is proved to be very effective. The main individual stages of the "Stage gate process" are shown in figure 2: 


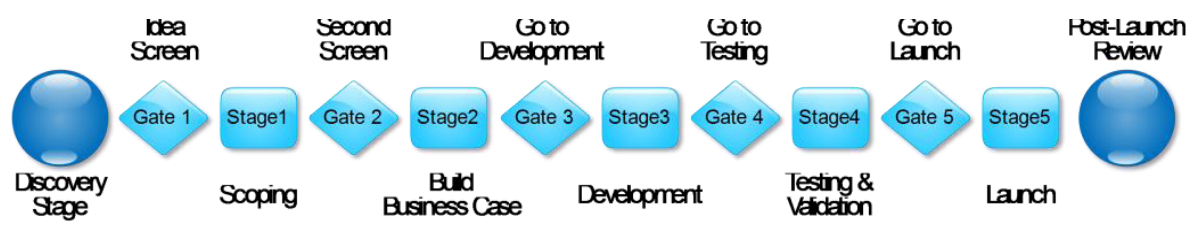

Fig. 2. Stage-Gate Process

\section{Cultural Product Design Based on Consumer Cognition}

According to three levels of consumers' cognition on cultural products and product design procedures, cultural product design based on consumers' cognition can be divided into research and analysis of consumers' cognition, image space establishment of cultural product cognition, cultural product concept design and implementation and cultural product test and evaluation. These four phases are shown in figure 3.

\subsection{Research and Analysis of Consumers' Cognition}

With certain designed object, designers, first of all, do research and analysis of the designed target consumers and carry out a comprehensive test and research on their countries, city, occupation, age, gender, educational level, marital status, income, family income and other aspects. Second, they carry out in-depth investigations and studies in cognitive experience of culture products from the perspective of the three levels : interpretation, experience and cognition.

\subsection{Image Space Establishment of Cultural Product Cognition}

According to the analysis of cognitive three level contents of target consumers, the designer pursue product cultural design elements from the three aspects of culture, people, and goods environment.

Designers collate these collected cultural design elements, on which target consumer groups design cognitive tests so as to establish image space of product culture.

\subsection{Cultural Product Concept Design and Implementation}

The main work of this stage is conversion, application, and integration of cultural elements, which makes the design process more clear, and systematic. Designers can achieve implantation of cultural concept and draw concept sketches through design semantics, and pictograph, self-explanatory, ideographic, and phonetic in semiotics.

Then the designers test and evaluate concept sketches early through people, color, material, process, function, structure, environment and other aspect, finally choose the best design sketch and form concept model scheme through CAD or the traditional design performance. At the same time, the best design concept model selection scheme of a certain amount of cognitive test by the target consumer groups, to determine whether the design of the products meet three hierarchy needs of consumers' cognition. Qualified products will next be put into three dimensional model making, 
and those not accord with the demand will be tested again after the selection or improved design.

They select a certain amount of best design concept model scheme, which is to be under cognitive test by target consumer groups to make sure whether the design of products meets three hierarchy needs of consumers' cognition.

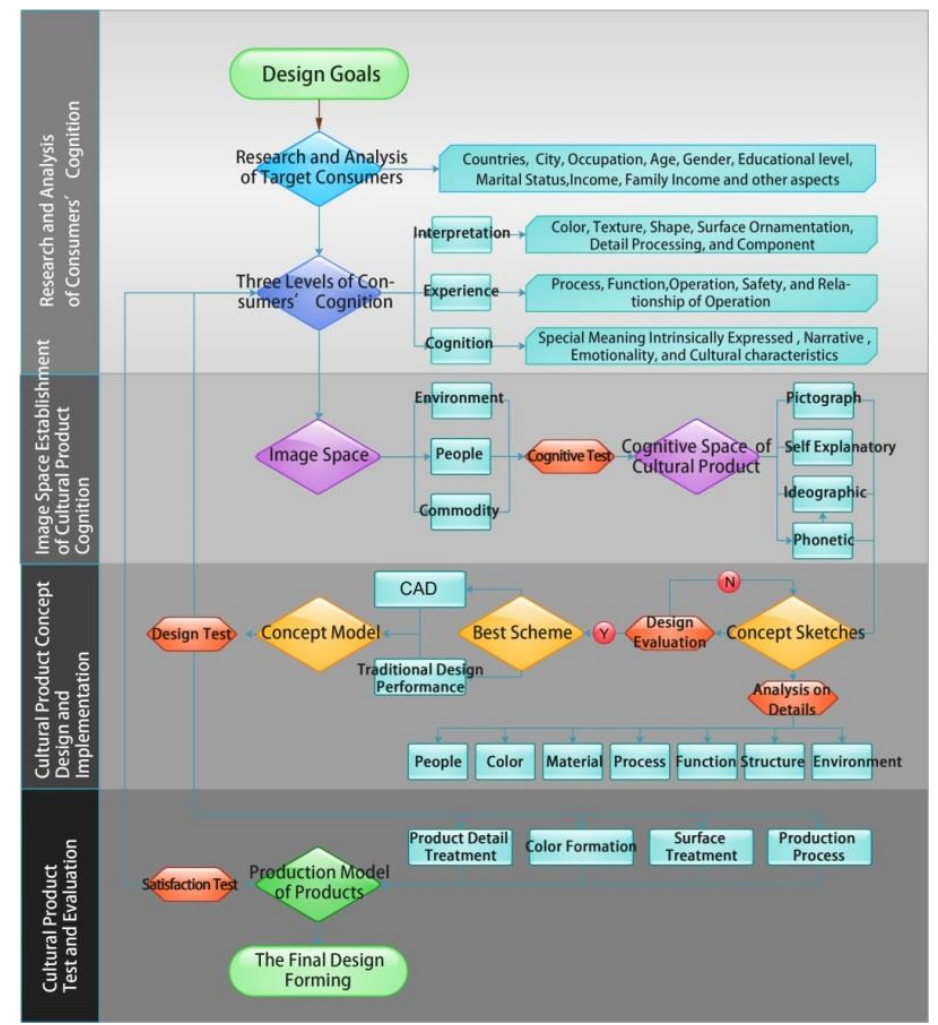

Fig. 3. Cultural Product Design Based on Consumer Cognition

\subsection{Cultural Product Test and Evaluation}

Designers produce models through 3D software and they research product detail treatment, color formation, surface treatment, and production process through produced model ones. Production model of products to meet consumers' cognition is made after repeated scrutiny and verification. The final production model passes customer satisfaction testing, thus the final design forming.

\section{$5 \quad$ Conclusion and Suggestion}

This paper makes a deep intensive study of design method and model of cultural product design based on consumers' cognition according to Psychology, Consumer 
Psychology, Cognitive Psychology, Mental Engineering, Ergonomics, Design Semantics, Semiotics and other theories. According to human cognitive system in the cognitive psychology, it proposes consumers' interpretation ,experience, and cognition ,these three hierarchy theory on cultural products, and raises cultural product design process based on consumers' cognition on the basis of the theory, which provides effective research methods and research model for the future study of cultural product design based on consumers' cognition, and also provides a foundation for further research and development of this issue.

The designer can further the study based on the research method and model, proposes different evaluation, design and test methods, further design practice according to different cultural products.

\section{References}

1. Xu, Y.: The modern design of the cultural connotation. Journal of Nantong University Social Sciences Edition (4), 98 (2007)

2. Zhang, M.-S.: Research on Product Semantics Based on the Cognitive and Psychological Factors, pp. 8-12. Tianjin Polytechnic University, Tianjin (2007)

3. Lin, W.: Fusion Research on China Elements of Traditional Culture and Modern Design, pp. 6-12. Jiangnan University, Wuxi (2008)

4. Peng, Y.: Research on the Process of Product Innovation System of Industrial Design, pp. 13-15. Sichuan University, Chengdu (2006)

5. Li, S.-Y., Shi, J.: Impact on Consumer Brand Preference for Cultural Products demand. Enterprise Economy (3), 10-12 (2013)

6. Wang, Z.-G.: Comparison and Innovation of Product Design Process. Packaging Engineering 25(2), 154-159 (2004)

7. Desmet, P.M.A., Overbeeke, C.J., Tax, S.J.E.T.: Designing products with added emotional value. In: Conference on Very Large Data Bases, Dublin, pp. 134-145 (1993)

8. Cheng, J., Zhou, M., Ye, J.: The Study of Modern Emergency Products under the Direction of New Ergonomics. In: Rau, P.L.P. (ed.) CCD/HCII 2013, Part I. LNCS, vol. 8023, pp. 31-40. Springer, Heidelberg (2013) 